\title{
Progression of Visual Acuity and Fundus Autofluorescence in Recent Onset Stargardt Disease: ProgStar Study Report \#4
}

Xiangrong Kong, $\mathrm{PhD}^{1}$

Sheila K West, $\mathrm{PhD}^{2}$

Rupert W Strauss' MD ${ }^{3,4,5,6}$

Beatriz Munoz, $\mathrm{MS}^{2}$

Artur V Cideciyan, $\mathrm{PhD}^{7}$

Michel Michaelides, $\mathrm{MD}^{5,6}$, Alexander $\mathrm{Ho}^{8}$, $\mathrm{BSC}$

Mohamed Ahmed ${ }^{3}$, MD

Etienne M Schönbach, $\mathrm{MD}^{3}$

Janet K Cheetham, PharmD ${ }^{9}$

Ann M Ervin, $\mathrm{PhD}^{1}$

Hendrik PN Scholl, MD, $\mathrm{MA}^{10}$.

on behalf of the ProgStar study group.

1. Bloomberg School of Public Health, Johns Hopkins University, Baltimore, Maryland

2. Dana Center for Preventive Ophthalmology, Wilmer Eye Institute, Johns Hopkins University, Baltimore, Maryland

3. Wilmer Eye Institute, Johns Hopkins University

4. Department of Ophthalmology, Johannes Kepler University Linz, Linz, Austria

5. Moorfields Eye Hospital, London, UK

6. Institute of Ophthalmology, University College London, London, UK

7. Scheie Eye Institute, Perelman School of Medicine, University of Pennsylvania, Philadelphia, Pennsylvania

8. Doheny Image Reading Center, Doheny, University of California, Los Angeles, California

8. Foundation Fighting Blindness, Columbia, Maryland

10. Department of Ophthalmology, University of Basel, Basel, Switzerland

Keywords: Stargardt disease, visual acuity, fundus autofluorescence, structural and functional correlation, longitudinal study

Financial support: The ProgStar studies are supported by the Foundation Fighting Blindness Clinical Research Institute (FFB CRI) and a grant to FFB CRI by the U.S. Department of Defense USAMRMC TATRC, Fort Meade, Maryland (grant numbers W81-XWH-07-1-0720 
and W81XWH-09-2-0189); Hendrik Scholl is supported by the Shulsky Foundation, New York, NY; Ocular Albinism Research Fund (Clark Enterprises Inc.); unrestricted grant to the Wilmer Eye Institute from Research to Prevent Blindness; Baylor-Johns Hopkins Center for Mendelian Genetics (National Human Genome Research Institute, NHGRI/NIH; identification number: 1U54HG006542-01). Rupert W. Strauss is supported by the Austrian Science Fund (FWF; Project number: J 3383-B23). Sheila West is supported by Al Maghraby professorhip.

Financial disclosures: the author(s) have made the following disclosure(s):

Dr. Hendrik Scholl is a paid consultant of the following entities: Astellas Institute for Regenerative Medicine (AIRM); Boehringer Ingelheim Pharma GmbH \& Co. KG; Daiichi Sankyo, Inc.; Gerson Lehrman Group; Guidepoint; and Shire. Dr. Scholl is member of the Scientific Advisory Board of Gensight Biologics; Vision Medicines, Inc.; and Intellia Therapeutics, Inc. Dr. Scholl is member of the Data Monitoring and Safety Board/Committee of the following entities: Genentech Inc./F. Hoffmann-La Roche Ltd; Genzyme Corp./Sanofi, and ReNeuron Group Plc/Ora Inc. These arrangements have been reviewed and approved by the Johns Hopkins University in accordance with its conflict of interest policies. Johns Hopkins University and Bayer Pharma AG have an active research collaboration and option agreement. These arrangements have also been reviewed and approved by the University of Basel (Universitätsspital Basel, USB) in accordance with its conflict of interest policies. Dr. Hendrik Scholl is principal investigator of grants at the USB sponsored by the following entity: Acucela Inc.; NightstaRx Ltd.; QLT, Inc. Grants at USB are negotiated and administered by the institution (USB) which receives them on its proper accounts. Individual investigators who participate in the sponsored project(s) are not directly compensated by the sponsor but may receive salary or other support from the institution to support their effort on the project(s). Dr. Cheetham is a paid consultant for the Foundation Fighting Blindness, which funds this research.

Dr. Michel Michaelides is supported by grants from the National Institute for Health Research Biomedical Research Centre at Moorfields Eye Hospital National Health Service Foundation Trust and UCL Institute of Ophthalmology, Fight For Sight (UK), The Macular Society (UK), Moorfields Eye Hospital Special Trustees, Moorfields Eye Charity, the Foundation Fighting Blindness (USA), and Retinitis Pigmentosa Fighting Blindness. Dr. Michaelides is a recipient of an FFB Career Development Award.

Rupert W Strauss is supported by the Austrian Science Fund (Project J3383-B23) and the Foundation Fighting Blindness Clinical Research Institute.

Etienne Schönbach is supported by the Leopoldina Fellowship Program, German National Academy of Sciences, (Halle, Germany, Grant Number LPDS 2015-14).

Running head: Progression of Visual Acuity and Fundus Autofluorescence in Stargardt Disease 


\begin{abstract}
Purpose: To investigate the impact of areas of decreased fundus autofluorescence (AF) on visual acuity (VA) in molecularly confirmed Stargardt disease (STGD1) with recent symptom onset, and investigate the association between these structural and functional measures over time. Design: Prospective international, multi-center, observational study of Stargardt disease. Participants: 64 patients (124 eyes) aged $\geq$ six years at first study visit, with onset of symptoms $\leq 2$ years before the first visit.

Methods: AF images were graded for the presence and areas of definitely decreased AF (DDAF), questionably decreased AF (QDAF), and total decreased AF (DAF). First visit images were also graded for presence of these lesions and for the presence of increased AF in the fovea. VA was measured as best corrected or presenting acuity and converted to LogMAR. Crosssectional associations were measured using linear models with Generalized Estimating Equations. Longitudinal linear mixed effects models were used to estimate yearly progression rates of VA and AF lesion areas.

Main Outcome Measures: Rate of change in VA and rate of change of decreased AF area. Results: In cross-sectional analyses at baseline, VA was not significantly associated with area of DDAF ( $\mathrm{p}=0.86$ ), or QDAF ( $\mathrm{p}=0.11$ ), but was significantly associated with lesion involvement in the fovea $(\mathrm{p}<.001)$. The VA change rate was $0.054 \mathrm{LogMAR} / \mathrm{year}(\mathrm{p}<.001)$, and depended on initial level of VA (faster loss was observed in those with 20/30-20/70 at first visit, 0.114 LogMAR/year, 95\% CI=.090-.138). Growth of DDAF depended on the size of the lesion at first visit, with larger DDAF having faster growth. Regression of QDAF area over time was associated with significantly larger growth in DDAF $(\mathrm{p}<.001)$ suggesting that QDAF areas may lose further AF signal over time. The increase in area of DDAF, or total decreased AF, was not associated with change in VA ( $\mathrm{p}=0.62$, and $\mathrm{p}=0.27$, respectively).

Conclusions: In recent onset STGD1, the rate of VA loss was not significantly associated with the rate of increase in area of DDAF, QDAF, or DAF. For DDAF, the growth rate depended on the initial size of the lesion, a finding which will be helpful in stratifying these patients for intervention.
\end{abstract}




\section{Introduction}

Autosomal recessive Stargardt macular dystrophy (STGD1), caused by disease-causing sequence variants in the $A B C A 4$ gene, is the most common form of juvenile macular degeneration ${ }^{1,2}$. The mutation causes degeneration of the macular photoreceptors and the retinal pigment epithelium (RPE) initially. The natural history of STGD1, and determining the rate of progression using a variety of assessments, is of intense interest with the prospect of several clinical trials of new treatment options $\mathrm{s}^{3-5}$. Measures of progression can include structural measures such as abnormalities in retinal layers on optical coherence tomography, or primarily RPE abnormalities as demonstrated on fundus autofluorescence (AF) imaging; and functional measures such as visual acuity, or testing specific points of retinal function using microperimetry ${ }^{6}$. In this study, we determine the association of visual acuity (VA) with measures of retinal loss as determined by AF.

Data suggest that the progression of STGD1 is dependent on several factors, which may include the prior duration of disease symptoms at the point at which progression is to be measured ${ }^{7}$ and baseline parameters such as the RPE lesion area measured by $\mathrm{AF}^{8}$. Duration may thereby be a significant confounding factor in studying the natural history, or indeed confound the association between structural and functional measures. To mitigate this effect, we sought to evaluate the association of VA and AF in cases of recent onset Stargardt disease.

The ProgStar (Progression of atrophy secondary to Stargardt disease) study, the largest prospective natural history study of STGD1 to date, also includes patients with recent symptom onset, herein defined as less than two years. By assessing the loss of VA and changes in AF, and evaluating the association of VA with AF in this cohort, we aimed to better understand disease progression at the earliest stages when patients are likely to first present.

\section{Methods}

ProgStar is a natural history study of cases of STGD1, enrolled from 9 sites internationally. A detailed description of the study and the total population has been previously published ${ }^{6}$. In summary, there are two components of ProgStar, a retrospective chart review study of eligible patients, and a prospective cohort of patients who are currently being followed over time. The retrospective eligibility criteria were as follows: 1 ) patients (aged $\geq 6$ years) with $\geq 2$ pathogenic mutations in the $A B C A 4$ gene or 1 pathogenic mutation with a typical STGD1 phenotype; 2) In the opinion of the clinical investigator, there was a well-defined atrophic lesion at the most recent visit of at least $300 \mu \mathrm{m}$ in diameter; the total area of all lesions had to be $\leq 12 \mathrm{~mm}^{2}$. The presence of an atrophic lesion at the first retrospective visit was not required. The criteria were slightly different for the prospective study, in that eligible eyes had to have at least one welldemarcated area of atrophy as visualized by AF with a minimum diameter of $300 \mu \mathrm{m}$, and all 
lesions together had to add to less than or equal to $12 \mathrm{~mm}^{2}$; best-corrected VA had to be 20 ETDRS letters (20/400 Snellen equivalent) or better.

In ProgStar overall, 251 participants were enrolled in the retrospective study and 259 participants in the prospective study. For this study, we defined "recent onset" cases as the subset who selfreported onset of symptoms within the previous two years prior to their first ProgStar visit. To be eligible for these analyses, patients also had to have both VA data and a gradable AF image at the first visit. In the retrospective study, there were 36 recent onset patients who met such study criteria; contributing 69 eyes to the analyses. From the prospective study, 28 recent onset patients met study criteria, contributing 55 eyes to the analyses. Among the 124 study eyes, 118 had at least two visits with both AF and VA data which could be used for the longitudinal analyses

Ethics committee approval was provided by all participating centers' Institutional Review Boards and the Human Research Protection Office of the U.S. Army Medical Research \& Materiel Command. The studies have been registered at www.clinicaltrials.gov (Identifier NCT01977846). All patients gave informed consent prior to enrollment in the study for the prospective component, and for the retrospective component where the ethical boards required consent.

The chart abstraction of reported best corrected or presenting acuity was converted to LogMAR for the eyes from the retrospective study patients. For prospective study patients, best corrected ETDRS VA was measured during each study visit and converted to LogMAR. Chart review also recorded clinical exam results such as presence of RPE atrophy, or presence of flecks inside and outside the arcades

AF images were obtained and graded as previously described ${ }^{6}$. In brief, AF images were eligible if obtained with a Heidelberg Engineering® (Heidelberg, Germany) device such as Heidelberg Retina Angiograph $2^{\mathrm{TM}}$ (HRA2) or Heidelberg Spectralis ${ }^{\mathrm{TM}}$ (excitation light $488 \mathrm{~nm}$, barrier filter $500 \mathrm{~nm}$ ). These images were submitted to the Doheny Imaging Retina Center for grading. Using a standardized grading scheme as previously described ${ }^{6,9}$, the lesion areas were graded for definitely decreased AF (DDAF) and questionably decreased AF (QDAF). The total area of decreased AF (DAF) was then calculated as the sum of DDAF and QDAF. Images were also graded for the presence of a heterogeneous or homogeneous background. AF background uniformity was graded based on a previously published grading scheme: a homogeneous background (outside of lesion area) was defined as an even distribution of background autofluorescence; a heterogeneous background was defined as widespread small foci of increased or reduced autofluorescence ${ }^{8}$. The area of the respective lesions was semiautomatically evaluated using the RegionFinder module of the Heidelberg EyeExplorer ${ }^{\mathrm{TM}}$ (Heidelberg Engineering ${ }^{\odot}$, Heidelberg, Germany) with standard grading conventions ${ }^{10}$. Two certified graders independently reviewed the AF images. Adjudication processes were applied in 
cases of discordant gradings. If consensus could not be reached between two adjudicating graders, the final assessment was determined by a senior investigator. In addition, the baseline images were graded independently for the presence of a lesion, and type of lesion (DDAF, QDAF, or increased AF) in the foveal center point (Figure 1). Adjudication by a senior grader was obtained in cases of discordant gradings.

We collected age at enrollment, gender, race, and duration of symptoms at the first visit either from the medical record or by direct interview.

Patient demographic, and eye-level clinical and AF characteristics at the first visit were summarized. For VA specifically, baseline median and interquartile range was presented. Referencing the World Health Organization's International Classification of Diseases, 10th revision $^{11}$, VA was also categorized and tabulated as $\geq 20 / 30$ (LogMAR $\leq 0.18$ ) (i.e. no/minimal visual impairment [VI]), $<20 / 30$ to 20/70 (LogMAR 0.18-0.54) (i.e. mild VI), $<20 / 70$ to 20/200 (LogMAR 0.54-1.0) (i.e. moderate VI), and <20/200 (LogMAR 1.0-1.3) (i.e. severe VI or blindness). For the cross sectional associations between VA and AF lesion areas or other variables, linear models with Generalized Estimating Equations (GEE) were used.

Linear mixed effects models (LMM) were used on the longitudinal data to estimate the yearly progression rate of VA and lesion areas: the mean of VA or lesion areas was modeled as a linear function of time since baseline visit, with the intercept and slope parameters assumed to be normally distributed random effects. The LMM essentially estimated an average linear trend from all individuals, while the random effects allowed random variation of each individual's trajectory from the average trend. A compound symmetry covariance matrix was adopted for the measurement errors of the outcome variable. The model thus accounted for correlation between repeated measurements of the same eye and correlation between eyes of the same participant. The assumption of a linear trend was examined by graphical inspection. To further identify baseline variables (e.g. initial VA level or lesion size) associated with VA or AF lesion change rate, LMMs were used by including each variable and its interaction with time. Testing the coefficient of the interaction term infers whether the variable was associated with VA or AF lesion change rate. Additionally, use of the square root of AF lesion sizes was considered in the modeling. However, due to the difficulty in interpretation when modeling it with the outcome of VA, results from the original linear models were reported which showed reasonable model fit.

For the analysis estimating the associations between growth in AF lesion areas and change in VA during follow-up, the changes from the first visit to the last visit were first calculated for VA and lesion areas respectively, and linear models with GEE were then used to estimate the VA change associated with DDAF and total DAF growth. Additionally, a sub-analysis using linear models with GEE was conducted to examine whether the decrease in QDAF lesion area was associated with an increase in DDAF area, using eyes that showed decreased QDAF area by the last visit. 
All analyses were conducted in SAS 9.3, and two-sided p-values from Wald-tests were reported. All statistical tests used significance level alpha of 0.05 . For the cross-sectional analysis using GEE models, model fit was assessed using aggregated residuals, and for the longitudinal analysis using LMMs, model fit was assessed using plots of scaled residuals ${ }^{12}$, both of which was deemed reasonable.

\section{Results}

The sample for the cross-sectional analyses ( $\mathrm{N}=64,124$ eyes) was $59 \%$ female, with a median age of 22.5 years (Table 1). The median VA was 0.68 (Interquartile range $(I Q R)=0.22-0.88$ ) with $45 \%$ of the eyes having moderate impairment (VA worse than 20/70, up to 20/200) and $14.5 \%$ having severe impairment (VA worse than 20/200). In these recent onset cases, DDAF was present in $28.2 \%$ of eyes ( $\mathrm{N}=35$ eyes), and the mean area in these eyes was $1.76 \mathrm{~mm}^{2}$. A total of 107 eyes $\left(86.3 \%\right.$ ) had QDAF, and the mean area was $1.17 \mathrm{~mm}^{2}$ (Table 1).

\section{Cross-sectional analysis}

The mean acuity of those eyes with DDAF was 0.60 at baseline (20/80 Snellen equivalent), and those with QDAF was 0.61 LogMAR. There were too few eyes with no lesion at baseline to reliably determine VA. Older age of onset was associated with better VA, and RPE atrophy found on clinical exam was associated with worse VA at first visit. Presence of an AF lesion in the fovea was associated with worse VA (Table 2): compared with no lesion in the fovea, the presence of DDAF in the fovea was associated with a worse VA of 0.52 LogMAR ( $~ 5$ lines), and the presence of QDAF in the fovea was associated with a worse VA of 0.31 ( 3 lines). Increased $\mathrm{AF}$ in the fovea was associated with a worse VA of $0.35(\sim 3.5$ lines), but the association was not statistically significant. These associations can be seen in the boxplots in Figure 2 showing the distributions of VA by AF lesion type in the fovea. However, larger areas of DDAF, QDAF and total DAF were not significantly associated with worse VA (Table 3).

\section{Longitudinal analysis: AF area change over follow-up}

The median follow-up time for the longitudinal component of the analyses was 1 year, with an IQR of 1-3.2 years. The rate of change over time in area of DAF depended both on the lesion type and the initial size. For DDAF, the average rate of area growth was $0.39 \mathrm{~mm} 2 /$ year, $(95 \%$ $\mathrm{CI}=.28-.51, \mathrm{p}<.001$ ) (Figure 3A), but the growth rate depended on the initial size of the lesion at first visit: for each $1 \mathrm{~mm}^{2}$ larger in lesion size at the first visit, the rate of growth was $0.37 \mathrm{~mm}^{2}$ larger per year $(\mathrm{p}<.001)$. For QDAF, the average increase was $0.11 \mathrm{~mm}^{2} /$ year $(95 \% \mathrm{CI}=.05-0.16$ $\left.\mathrm{mm}^{2}\right)(\mathrm{p}<.001)$ (Figure 3B). Interestingly, over time in 32 eyes, the QDAF lesion decreased. For those eyes that lost QDAF, we hypothesized that there would be a corresponding increase in DDAF. Analysis showed the association was significant, that for every $1 \mathrm{~mm}^{2}$ decrease in 
QDAF, there was a corresponding growth in DDAF area of $0.45 \mathrm{~mm}^{2}\left(95 \% \mathrm{CI}=0.29-0.61 \mathrm{~mm}^{2}\right)$, $\mathrm{p}=<.001$.

\section{Longitudinal analysis: VA change over follow-up}

The overall rate of change in VA was $0.054 \operatorname{LogMAR} /$ year $(\mathrm{p}=<.001)$, equivalent to about $1 / 2$ of a Snellen line per year, but the rate of change depended on the starting level of VA. (Figure 4a-d). The faster rate of VA loss occurred among eyes with no or mild visual impairment at first visit, slowed for eyes with moderate visual impairment, and actually improved for those whose starting vision was worse than $20 / 200$.

We incorporated VA at first visit in models examining the impact of other predictors on rate of change in VA over time. Table 4 shows the baseline characteristics that were examined for an association with greater or lesser rate of change in VA. Older age at baseline (or older age of symptom onset) was significantly associated with slower VA change after adjusting for baseline VA level. Baseline size of QDAF was not associated with VA change rate. The baseline size of the area of DDAF and total DAF was associated with the rate of change VA in unadjusted analysis, but such association was not significant after adjusting for baseline VA level. Eyes without any lesion in the fovea at baseline had a mean VA loss rate of $0.066 \operatorname{LogMAR} /$ year; eyes with QDAF lesion in the fovea had a VA loss rate of .031 LogMAR/year; eyes with DDAF in the fovea had a non-significant loss rate of .004 LogMAR /year; and eyes with increased AF in the fovea also had a non-significant loss rate of.031 LogMAR/year. However, once adjusted for baseline VA status, the presence of DDAF and QDAF, compared to having no lesion in the fovea, was significantly associated with a greater rate of loss of VA, the difference in rate of loss of VA being 0.102 and .047 LogMAR/year respectively. Clinically observed flecks inside the arcades at baseline was not associated with VA change. Clinically observed flecks outside the arcades, or having a heterogeneous background on AF image had slower rate of change in VA than those with a homogeneous background or no flecks outside the arcades.

\section{Longitudinal analysis: AF change vs. VA change over follow-up}

We hypothesized that a faster decline in VA would be predicted by a faster growth in DDAF or DAF area over the follow up period. However, we did not find that the change in VA over the follow-up period was significantly associated with the growth in area of DDAF, or DAF, alone or adjusted for the baseline level of VA (Table 5 and Figure 5). (Figures 6 and 7 in supplemental materials show the overall change in DAF area, and overall change in VA. Available at aaojournal.org)

\section{Discussion}

We presented data of a cohort of STGD1 patients derived from ProgStar with recent onset of symptoms, and assessed the association of vision, as estimated by VA, and presence of RPE 
lesions, as demonstrated by AF. Assessment in such a cohort minimized any potential confounding due to differing duration of symptoms in the patients.

Of note, even in this group of patients with onset of symptoms within the last two years, with a median age of just 22.5 years, more than half the sample of eyes had acuity worse than 20/70 at baseline and $94 \%$ had DAF in their images already. However, almost $3 / 4$ of the eyes were graded as not having a heterogeneous background in their images, and close to $60 \%$ did not have flecks outside the arcades through clinical observation. The clinical picture of these recent onset cases is one of a sizeable number with poor vision and DAF in the images, but not disseminated disease in terms of heterogeneous background or flecks outside the arcades. This baseline is critical to understanding the risk of losing vision in patients reporting recent onset of symptoms.

We observed a faster rate of VA loss associated with a homogeneous background at baseline, compared to a heterogeneous background. The rate of VA loss was low for those with heterogeneous backgrounds (.017 LogMAR/year), but in general, less than $1 / 4$ of eyes in these new onset presented with heterogeneous background. We also found faster progression of VA associated with younger age (i.e. with earlier onset of symptoms). This finding conforms to other studies and to our analysis with the overall ProgStar retrospective cohort ${ }^{13,14}$.

At baseline, $20 \%$ eyes had no lesion in the fovea. A similar proportion was reported previously for STDG1 patients with foveal sparing phenotype ${ }^{15,16}$. In our cohort, however, eyes with no foveal lesion could include both eyes with foveal sparing (AF lesion encircling the fovea $\geq 180^{\circ}$ ) and eyes with extrafoveal (but not fovea encircling) lesion. We found cross sectional associations between the presence of DAF lesions (DDAF or QDAF) in the fovea, and worse vision at baseline. The presence of increased AF in the fovea was also associated with worse vision with a point estimate similar to QDAF, but was not statistically significant likely due to small numbers with this lesion type in the fovea. Longitudinally, adjusted for baseline acuity, the rate of loss of acuity was greater in eyes with QDAF and DDAF in the fovea at baseline compared to eyes that did not have these lesions. This finding is logical, as high visual acuity depends on anatomical integrity of the foveal center and acuity is worse if measured using an eccentric locus ${ }^{17,18}$. Others have also found that loss of VA over time is slower in those with no lesion in the fovea at the outset ${ }^{19}$. It is known that foveal sparing phenotype is on the milder end of the spectrum of STGD1 and that VA of STGD1 patients with foveal sparing can be well-maintained till later stage $^{16,20,21}$. In our analysis, however, the rate of VA change in eyes with no foveal lesion at baseline was faster (not statistically significant) than eyes with increased AF at baseline. This faster rate in eyes with no foveal lesion could be driven by those eyes with non fovea encircling lesion. We are limited to test this given that our grading did not differentiate between eyes with foveal sparing and eyes with non fovea encircling lesion. 
Unlike to previously reported, ${ }^{22}$ we did not find significant associations between AF lesion growth and VA decline. This finding is supported by: first, we found no statistically (or clinically) significant association cross-sectionally of worse vision with the larger area of DDAF, QDAF, or total DAF lesion at baseline; second, longitudinally, with larger area of QDAF or DDAF at baseline, we found a slower rate of change in visual acuity over time, but once adjusted for baseline acuity, there was no significant difference in the rate of change in acuity with larger size of either lesion; third, in modeling the area of growth in DDAF or total DAF over time, we found no significant association of larger lesion growth with greater change in VA during the time. These all suggest that faster lesion growth may not be associated with faster rate of worsening vision. We were hampered in further characterizing these associations by the absence of data on the location of the DAF lesion at baseline (except in the fovea), and the direction of growth, especially into the fovea or into an eccentric locus. Thus, if the lesion area is large or growing, but remains sparing the fovea or the eccentric locus, there may be no or modest change in vision. Alternatively, small lesions that are in the foveal center will correlate with poor vision at the outset, and further growth may not greatly impact the already poor vision. Another explanation may be that the relatively small rate of change in QDAF $\left(0.11 \mathrm{~mm}^{2} /\right.$ year $)$ and DDAF (on average $0.67 \mathrm{~mm}^{2}$ ) may not be enough to hasten visual acuity loss in these new onset cases. Others have found a lack of association of acuity with growth in DAF lesions as well ${ }^{23}$. Finally, our follow-up period was on average one year, with an interquartile range of 3.2 years; this interval may not have been sufficient to observe an impact of growth of DAF on change in visual acuity. If so, then the functional consequences of growth in DAF in a relatively short period is very low.

For recent onset cases, the rate of increase in area of DAF depended on whether it was DDAF or QDAF, and for DDAF, the initial size of the lesion. As expected, the initial lesion size was smaller than the full patient population enrolled in ProgStar ${ }^{6}$. Our rate of growth for lesions of that size is consistent with the rate in other studies, where the initial lesion size was not reported as a factor for progression $8,23,24$. For QDAF, the overall growth rate for the recent onset cases was $0.11 \mathrm{~mm}^{2} /$ year, but decrease on QDAF area was also observed in 32 eyes, with associated increase in DDAF over the same time. This may reflect that QDAF converts to new areas of DDAF. This dynamic process is also supported by the fact that in our recent onset cases, at first visit most of the DAF was taken up with eyes that had QDAF (occurring in 107 of the 117 eyes with DAF).

Longitudinally, we expected an association of a faster rate of growth of the area of DAF with a faster loss of VA, but this was not the case. The point estimates for the association were close to zero and not statistically significant. Several issues may affect this lack of association. Most importantly, it is likely critical to not only chart the growth in area, but the location of growth and whether the growth affected the foveal center or the preferred retinal locus (PRL). We would expect the association should be observed for those cases. Another issue is the possible 
improvement in vision over time, which as discussed above, could reflect better adjustment to a PRL, even in the face of lesion growth as long as the growth is not in the PRL. Others have also found that acuity is not well associated with DAF growth over time, although lesion sizes were not reported in that study ${ }^{23}$. A third issue is, as mentioned before, growth of DAF in the relatively short duration of follow-up for these recent onset cases may not be large enough to significantly worsen VA.

In recent onset cases, the rate of change in vision depended on vision at first visit, with worse rate of loss in those starting with mild or no vision impairment. The rates are very similar to those observed in the overall ProgStar retrospective cohort, although those with much longer duration of symptoms presented with worse vision at first visit and tended to have a slower rate of change ${ }^{14}$. In the few recent onset patients who presented with very poor vision (VA>20/200), there was actual improvement in visual acuity of $0.04 \mathrm{Log}$ MAR/year. This same trend was found in the overall ProgStar retrospective cohort, where the improvement in VA for those with poor VA at first visit was at a rate of $0.05 \log M A R / y e a r^{14}$. The improvement for this group is very modest, less than half a line. It might reflect regression to the mean, where patients with poor vision tested poorly at baseline, and tested slightly better at subsequent visits reflecting normal variation. Improvement may also reflect adjustment to change in $\mathrm{PRL}^{25}$. This latter explanation may be especially valid in the case of recent onset cases, whose onset was sudden with dramatic loss of vision at early visits, with adjustment over time. We do not have microperimetry on the retrospective ProgStar patients to determine if eccentric fixation at first visit was present.

In summary, our study of patients with recent onset Stargardt disease type 1 has found overall a small rate of VA loss per year, dependent on the level of VA at first visit. The worse VA at first visit was associated with presence of QDAF and DDAF in the fovea, but not the size of DAF lesions. Loss of VA over time was slower in those with larger DDAF or QDAF lesions, but not significantly slower once adjusted for baseline acuity levels. However, rate of loss of acuity was significantly worse with DAF in the fovea at baseline compared to no foveal lesion. The growth in DAF, and DDAF was not found to be associated with change in VA, suggesting that the location of lesion growth may be more important in determining the consequences for acuity than the physical AF lesion growth itself in patients with recent symptom onset. 
References

1. Michaelides M, Hunt DM, Moore AT. The genetics of inherited macular dystrophies. Journal of medical genetics. Sep 2003;40(9):641-650.

2. Allikmets $\mathrm{R}$, Singh $\mathrm{N}$, Sun $\mathrm{H}$, et al. A photoreceptor cell-specific ATP-binding transporter gene $(A B C R)$ is mutated in recessive Stargardt macular dystrophy. Nature genetics. Mar 1997;15(3):236-246.

3. Schwartz SD, Regillo CD, Lam BL, et al. Human embryonic stem cell-derived retinal pigment epithelium in patients with age-related macular degeneration and Stargardt's macular dystrophy: follow-up of two open-label phase 1/2 studies. Lancet. Feb 7 2015;385(9967):509516.

4. Dalkara D, Goureau O, Marazova K, Sahel JA. Let There Be Light: Gene and Cell Therapy for Blindness. Human gene therapy. Feb 2016;27(2):134-147.

5. Thompson DA, Ali RR, Banin E, et al. Advancing therapeutic strategies for inherited retinal degeneration: recommendations from the Monaciano Symposium. Investigative ophthalmology \& visual science. Feb 2015;56(2):918-931.

6. Strauss RW, Ho A, Munoz B, et al. The Natural History of the Progression of Atrophy Secondary to Stargardt Disease (ProgStar) Studies: Design and Baseline Characteristics: ProgStar Report No. 1. Ophthalmology. Apr 2016;123(4):817-828.

7. Cideciyan AV, Swider M, Aleman TS, et al. ABCA4 disease progression and a proposed strategy for gene therapy. Human molecular genetics. Mar 1 2009;18(5):931-941.

8. Fujinami K, Lois N, Mukherjee R, et al. A longitudinal study of Stargardt disease: quantitative assessment of fundus autofluorescence, progression, and genotype correlations. Investigative ophthalmology \& visual science. Dec 2013;54(13):8181-8190.

9. Strauss RW, Munoz B, Jha A, et al. Comparison of short-wavelength reduced-illuminance and conventional autofluorescence imaging in Stargardt Macular Dystrophy. American journal of ophthalmology. Jun 102016.

10. Schmitz-Valckenberg S, Brinkmann CK, Alten F, et al. Semiautomated image processing method for identification and quantification of geographic atrophy in age-related macular degeneration. Investigative ophthalmology \& visual science. Sep 2011;52(10):7640-7646.

11. Johnson GJM, Darwin C. ; Weale, Robert A. ; West, Sheila K. The Epidemiology of Eye Disease. 3 ed: World Scientific Publishing Company; 2012.

12. Lin DY, Wei L, Ying Z. Model-checking techniques based on cumulative residuals. Biometrics. Mar 2002;58(1):1-12.

13. Cideciyan AV, Swider M, Schwartz SB, Stone EM, Jacobson SG. Predicting Progression of ABCA4Associated Retinal Degenerations Based on Longitudinal Measurements of the Leading Disease Front. Investigative ophthalmology \& visual science. Sep 2015;56(10):5946-5955.

14. Kong X; Strauss RW; Michaelodes M; Cidaciyan A; Sahel J-A; Munoz B; West SSH. Visual Acuity Loss and Associated Risk Factors in the retrospective Progression of Stargardt disease Study (ProgStar report No.2). Ophthalmology. 2016.

15. Lambertus S, Lindner M, Bax NM, et al. Progression of Late-Onset Stargardt Disease. Investigative ophthalmology \& visual science. Oct 01 2016;57(13):5186-5191.

16. van Huet RA, Bax NM, Westeneng-Van Haaften SC, et al. Foveal sparing in Stargardt disease. Investigative ophthalmology \& visual science. Oct 16 2014;55(11):7467-7478. 
17. Messias A, Reinhard J, Velasco e Cruz AA, Dietz K, MacKeben M, Trauzettel-Klosinski S. Eccentric fixation in Stargardt's disease assessed by Tubingen perimetry. Investigative ophthalmology \& visual science. Dec 2007;48(12):5815-5822.

18. Reinhard J, Messias A, Dietz K, et al. Quantifying fixation in patients with Stargardt disease. Vision research. Jul 2007;47(15):2076-2085.

19. Rotenstreich $Y$, Fishman GA, Anderson RJ. Visual acuity loss and clinical observations in a large series of patients with Stargardt disease. Ophthalmology. Jun 2003;110(6):1151-1158.

20. Fujinami K, Sergouniotis PI, Davidson AE, et al. Clinical and molecular analysis of Stargardt disease with preserved foveal structure and function. American journal of ophthalmology. Sep 2013;156(3):487-501 e481.

21. Westeneng-van Haaften SC, Boon CJ, Cremers FP, Hoefsloot LH, den Hollander Al, Hoyng CB. Clinical and genetic characteristics of late-onset Stargardt's disease. Ophthalmology. Jun 2012;119(6):1199-1210.

22. Testa F, Melillo P, Di lorio V, et al. Macular function and morphologic features in juvenile stargardt disease: longitudinal study. Ophthalmology. Dec 2014;121(12):2399-2405.

23. McBain VA, Townend J, Lois N. Progression of retinal pigment epithelial atrophy in stargardt disease. American journal of ophthalmology. Jul 2012;154(1):146-154.

24. Chen B, Tosha C, Gorin MB, Nusinowitz S. Analysis of autofluorescent retinal images and measurement of atrophic lesion growth in Stargardt disease. Experimental eye research. Aug 2010;91(2):143-152.

25. Tarita-Nistor L, Gonzalez EG, Markowitz SN, Steinbach MJ. Plasticity of fixation in patients with central vision loss. Visual neuroscience. Nov 2009;26(5-6):487-494. 
Figure legends:

Figure 1. Autofluorescence image showing different lesions from 4 different eyes with Stargardt disease. Image A shows foveal sparing disease with multifocal extrafoveal lesions; the yellow, green, and white arrows show DDAF lesions, QDAF lesions, and normal foveal area, respectively. Image B shows well-demarcated QDAF lesion (orange arrow) involving the foveal center surrounded by an area of poorly-demarcated QDAF lesion (green arrow). Image C shows a large DDAF lesion (yellow arrow) involving the foveal center surrounded by an area of poorlydemarcated QDAF lesion (green arrow). Image D shows hyper-autofluorescent area involving the foveal center (white arrow) surrounded by another area of QDAF (green arrow) with a ring of edge hyper-autofluorescence.

Figure 2: Boxplots showing the distributions of visual acuity (VA) at the first study visit ( $\mathrm{N}=124$ eyes) by fundus autofluorescence (AF) lesion findings at the foveal center. In each boxplot, the lower and upper boundary of the box represents the first quartile (i.e. 25th percentile) and the third quartile (i.e. 75th percentile) of the data, respectively; the horizontal black line in the box is the median; the black diamond is the mean; the lower and upper whiskers are the lowest and highest values (Nobs: number of eyes (observation) in the group).

Figure 3: Spaghetti plots showing the change over time in definitely decreased autofluorescence (DDAF) area and questionably decreased autofluorescence (QDAF) area ( $\mathrm{N}=118$ eyes) in recent onset Stargardt. Each line shows data from one eye.

3A. Spaghetti plot for DDAF area change.

3B. Spaghetti plot for QDAF area change. ( $N=32$ eyes had decreased QDAF over the follow-up period.)

Figure 4: Spaghetti plot showing visual acuity change from baseline VA level. Each line shows VA data of one eye over the follow-up period.

4A: The rate of change in Visual Acuity in eyes with no or mild visual impairment at first visit (i.e. VA was $20 / 30$ or better)*

4B: The rate of change in Visual Acuity in eyes with moderate visual impairment at first visit (VA was <20/30 to 20/70)*

4C. The rate of change in Visual Acuity in eyes whose acuity at first visit was $<20 / 70$ 20/200*

4D: The rate of change in Visual Acuity in eyes with visual acuity at first visit <20/200*

Figure 5. Scatter plots of the area of growth of DDAF (panel A) and total decreased autofluorescence (DAF; panel B) vs. the change of VA between the first and last visits. 